\title{
Biliary tract cancers: systemic therapy for advanced disease
}

\author{
Francisco J. Martinez, Rachna T. Shroff \\ Division of Hematology/Oncology, University of Arizona Cancer Center, Tucson, AZ, USA \\ Contributions: (I) Conception and design: All author; (II) Administrative support: All author; (III) Provision of study materials or patients: All authors; \\ (IV) Collection and assembly of data: All authors; (V) Data analysis and interpretation: All authors; (VI) Manuscript writing: All authors; (VII) Final \\ approval of manuscript: All authors. \\ Correspondence to: Rachna T. Shroff, MD, MS. Chief, Section of GI Medical Oncology Associate Professor of Medicine, Division of Hematology/ \\ Oncology, University of Arizona Cancer Center, 1515 N. Campbell Ave., Tucson, AZ 85724, USA. Email: rshroff@email.arizona.edu.
}

\begin{abstract}
Biliary tract carcinomas (BTC) present with minimal symptoms and thus they are often diagnosed in advanced stages that require systemic therapy. Unfortunately, if not resected, BTC's prognosis is generally poor, in part due to limited therapeutic options. Herein we will highlight the various systemic therapies that have proven efficacy in these diseases in both $1^{\text {st }}$ and $2^{\text {nd }}$ line. As it stands now, the combination of Gemcitabine and Cisplatin is the gold standard. Gemcitabine plus Cisplatin (Gem-Cis)-nab-paclitaxel showed improved survival in a phase II trial compared with historical controls. SWOG 1815 is a phase III trial currently underway comparing Gem-Cis-nab-paclitaxel to Gem-Cis and if positive, this has the potential to establish a new standard of care. New data from the ABC-06 study has shown a survival benefit using FOLFOX in the $2^{\text {nd }}$ line setting. Molecularly targeted agents in BTC have demonstrated potential beyond Gem-Cis and while currently limited to second- and later-line therapies, ongoing trials are testing their efficacy even in newly diagnosed patients. With both incremental improvements in existing therapies and the development of entirely novel agents, the future of systemic therapy for BTC is promising.
\end{abstract}

Keywords: Biliary tract carcinomas (BTC); systemic therapy; gemcitabine-cisplatin; FOLFOX; review

Submitted Aug 03, 2019. Accepted for publication Dec 05, 2019.

doi: $10.21037 /$ cco.2019.12.07

View this article at: http://dx.doi.org/10.21037/cco.2019.12.07

Biliary tract carcinomas (BTC) are defined as carcinomas of the intrahepatic bile ducts, extrahepatic bile ducts or gall bladder (1). They present with minimal symptoms and thus they are often diagnosed in advanced stages that require systemic therapy. Unfortunately, if not resected, BTC's prognosis is generally poor in part due to limited therapeutic options. In this article, we will review the various chemotherapies that have proven efficacy in these diseases, while additionally discussing the promising literature surrounding other novel therapeutic approaches.

Gemcitabine plus Cisplatin (Gem-Cis) chemotherapy is the gold standard in the treatment of BTCs. Valle and colleagues showed the benefits of Gem-Cis vs. Gemcitabine alone in a multicenter, phase III, randomized control trial. Patients on the combination arm demonstrated a median overall survival of 11.7 months compared to 8.1 months with single-agent gemcitabine (2). The ABC-02 trial in
2010 was a landmark study as it was the first randomized phase III trial in BTC and established a new chemotherapy backbone for future trials. As it stands today, Gem-Cis has remained the standard of care for newly diagnosed patients with BTCs.

Since the ABC-02 trial, there have been a number of smaller trials combining Gemcitabine with other agents (3). An agent only approved in Asia, S-1 is an oral anticancer drug that consists of tegafur (FT) as a prodrug of 5-fluorouracil (5-FU), 5-chloro-2,4-dihydroxypyridine (CDHP), and potassium oxonate (Oxo). In a Phase 1 trial evaluating the addition of S-1 to cisplatin versus GemCis, the six-month PFS rates were $43.8 \%$ with Gem-Cis and $34.7 \%$ with S-1 plus cisplatin, [unadjusted HR (GP/ $\mathrm{SP})=0.85 ; 95 \% \mathrm{CI}, 0.52-1.36]$ (4). Understanding the side effect profile of nausea, vomiting requiring hydration before and after administration of Gem-Cis, Morizane 
et al. demonstrated in a phase III trial the noninferiority of using Gemcitabine with S-1. Three hundred and fifty-four patients were enrolled and Gemcitabine with S-1 showed a mOS of 15.1 months compared to the control of Gem-Cis of 13.4 months ( $90 \%$ CI, 0.78 to 1.15 ). The major advantage of this combination is that the hydration step is not needed thus making the logistics of administration easier.

There are scenarios in which the gold standard treatment can not be applied and alternative regimens pursued. One such common scenario is patients with renal dysfunction which can be a contraindication for Cisplatin. In 2008, André $e t a l$. evaluated the combination of gemcitabine with oxaliplatin (GEMOX) as first line therapy in advanced BTCs and showed mOS for the intent-to-treat population was 8.8 months (95\% CI, 6.9-11.1 months) (5). In 2010, Sharma et al. combined gemcitabine with oxaliplatin in a randomized control trial and compared it to fluorouracil (FU) and folinic acid (FA). They showed 4.6 months of PFS for the FUFA arm (95\% CI, 3-6.2 months), and 9.5 months for the mGEMOX arm (95\% CI, 5-14 months) (6). Later, a phase 3 randomized control trial combined Gemcitabine and Oxaliplatin with or without erlotinib, a tyrosine kinase inhibitor targeting the epidermal growth factor receptor (EGFR) pathway. This multicenter trial showed a median progression-free survival of 4.2 months (95\% CI, 2.7-5.7 months) in the chemotherapy alone group and 5.8 months (95\% CI, 4.6-7.0 months) in the chemotherapy plus erlotinib group (95\% CI, 0.61-1.03), however median overall survival was the same in both groups-9.5 months (95\% CI, 7.5-11.5 months) in the chemotherapy alone group and 9.5 months (95\% CI, 7.611.4 months) in the chemotherapy plus erlotinib group (7). Gemcitabine has also been combined with capecitabine, an oral fluoropyrimidine, in a single-arm phase II clinical trial where the patients had pathologically proven advanced biliary tract carcinoma with no prior chemotherapy. This study demonstrated a median progression-free survival of 7 months (95\% CI, 4.6-11.8 months) (8). While these studies do not provide head-to-head comparisons with Gem-Cis, their efficacy appears in line with historical controls, suggesting that the combinations of GEMOX or Gem-Cape could be considered as alternatives for patients who were cisplatin ineligible.

Another common scenario in advanced BTC is hyperbilirubinemia despite stenting, thus preventing the safe admistration of gemcitabine. In 2019 in a multicentered open label phase III studied capecitabine plus oxaliplatin (XELOX) against GEMOX and showed a mOS time of
10.4 months with GEMOX (95\% CI, 8.0-12.6 months) and 10.6 months (95\% CI, 7.3-15.5 months) in the XELOX group (9). While still inferior to historical controls of Gem-Cis, this regimen does offer a therapeutic option for patients unable to safely receive gemcitabine chemotherapy.

Thus far, no other combination agent has been able to supplant Gem-Cis, however a triplet combination of Gemcitabine, Cisplatin, and nab-paclitaxel has shown promising results. In a single-arm phase II multicenter clinical trial, 60 patients with advanced biliary tract cancer received the above three-drug combination. The trial reached its primary endpoint with a median progressionfree survival of 11.8 months (95\% CI, 6.0-15.6 months), which, when compared to the historical control of Gem-Cis (mPFS of 8 months), was felt to be clinically meaningful. More striking, the mOS was 19.2 months (95\% CI, 13.2 months to not estimable) which was a marked improvement from the historical ABC-02 mOS data of 11.7 months (10). An additional component of the trial was comparing the triplet therapy at a reduced dose to avoid toxicities. The trial showed no difference in efficacy at the reduced dose and found that it was relatively well tolerated at the adjusted doses used in the latter 30 patients enrolled. Gem-Cis-nab-paclitaxel is now being compared head-tohead against Gem-Cis in SWOG 1815, a randomized phase III trial, with 268 planned patients and a primary endpoint of mOS. If positive, Gem-Cis-nab-paclitaxel could be considered as a gold standard option in good performance status patients with newly diagnosed advanced biliary tract cancers.

Other combination therapies have seen success in pancreas cancer and are now being studied in advanced biliary tract cancer. The combination of 5 -fluorouracil, leucovorin, oxaliplatin, and irinotecan (FOLFIRINOX) has proven efficacy in metastatic pancreatic cancer (11). A phase II/III trial currently underway in France is based on the premise that pancreatic and bile duct cancers are biologically and, theoretically, prognostically similar (12). This regimen is currently being investigated head-to-head against GemCis in newly diagnosed patients with BTCs with the primary endpoint of mOS in the phase III portion (12). Three hundred and sixteen patients are planned to be enrolled and the results are eagerly anticipated.

While Gem-Cis remains the backbone for systemic therapy in newly diagnosed BTCs, until recently, there has not been a prospectively validated $2^{\text {nd }}$ line therapy for these patients. Retrospective data suggest dismal outcomes in the $2^{\text {nd }}$ line setting with response rates around $5 \%$ and median 
PFS in the $2-3$ month range $(12,13)$. Recently, Lamarca and colleagues presented their findings of $\mathrm{ABC}-06$, an openlabel, randomized, multicenter study comparing 12 cycles of FOLFOX (5-FU and oxaliplatin) with active symptom control (ASC) to ASC alone in the $2^{\text {nd }}$ line setting after GemCis. The ASC group did not receive any chemotherapy; only supportive care and palliative radiotherapy were allowed. The 81 patients randomized to the experimental arm (ASC + FOLFOX) had a median OS of 6.2 months and OS-rate of $25.9 \%$ at the 12 -month mark, while patients in the control arm demonstrated a median OS of 5.3 months and a 12 -month OS-rate of $11.4 \%$ (14). The benefit of FOLFOX was seen regardless of platinum sensitivity. While this positive study has now set the stage for a new standard of care for $2^{\text {nd }}$ line therapy, the absolute survival benefit is incremental, demonstrating a need for better options in refractory patients. Another separate retrospective review $(n=321)$ of second-line treatment after failure of Gem-Cis compared fluoropyrimidine monotherapy with a combination of fluoropyrimidine and a platinum-based agent (15). Fluoropyrimidine monotherapy was moderately effective [PFS =1.9 months (CI, 1.6-2.2 months), OS $=6.5$ months (CI, 5.7-7.0 months)], but the combination of FP and a platinum-based agent did not significantly improve PFS and OS; the combination did, however, improve response rate ( $1 \%$ vs. $8 \%, \mathrm{P}=0.009)$, demonstrating that combination treatment in second-line chemotherapy could be considered for those who can tolerate it.

Targeted therapy has been of great interest in oncology for many years, but previous studies only had limited success in BTCs. There are numerous trials looking at targeting the EGFR pathway with drugs including erlotinib, cetuximab, and panitumumab $(13,14)$. A meta-analysis conducted by Cai et al. analyzed 4 RCTs comparing GEMOX alone with GEMOX and the addition of an EGFR inhibitor (16). The addition of a targeted EGFR inhibitor to the regimen provided a benefit in PFS (HR $=0.80 ; 95 \% \mathrm{CI}, 0.66-0.94$, $\mathrm{P}=0.03)$ and correlated with a better ORR $(\mathrm{RR}=1.52 ; 95 \%$ CI, 1.13-2.04, $\mathrm{P} \leq 0.01$ ), though no clear overall survival benefit was noted. In addition to EGFR inhibitors, studies looking at blocking the vascular endothelial growth factor (VEGF) receptor pathway using bevacizumab, sorafenib, and cediranib have also been generally underwhelming (17). Morizane et al. conducted an extensive review on both the prevalence of potentially targetable driver mutations (including EGFR and VEGF overexpression) as well as success rates of phase II trials using the respective targeted therapies as monotherapy, in combination with cytotoxic agents, or in combination with other targeted therapies. In over 29 trials between 2006 and 2017, neither VEGF agents nor EGFR agents alone, in combination with each other, or in combination with other cytotoxic agents demonstrated success relative to first-line therapy. The authors did note that the lack of benefit could be attributed to these trials being done in unselected populations as opposed to utilizing biomarker-driven approaches.

With limited success of EGFR and VEGF targeted agents, the newer era of targeted therapy studies in BTCs has leveraged the number of potentially actionable mutations, with successful inhibition of the fibroblast growth factor receptor (FGFR) pathway in patients with FGFR2 fusions, blockade of isocitrate dehydrogenase (IDH) in patients with IDH1 and BRAF V600Emutations as key examples of targeted therapies in BTCs (16). In a multicenter case series $(\mathrm{n}=321)$, Javle et al. first defined the prevalence of genetic aberrations targetable by anticancer drugs among intrahepatic cholangiocarcinoma, extrahepatic cholangiocarcinoma, and gallbladder carcinoma, then examined if second-line targeted therapy (after standard first-line chemotherapy), along with other factors such as types of mutations and clinical variables (sex, age, tumor grade, pathological stage) correlated with OS (primary endpoint). They found significant prognostic implications among 3 types of mutations in IHCCA, with OS negatively impacted by the presence of TP53 and KRAS mutations $(\mathrm{P}=0.001$ and $\mathrm{P}<0.05)$ and positively impacted by the presence of FGFR2 alterations ( $\mathrm{P}=0.001)$ (18). Additionally, patients with FGFR2 fusions in particular who were treated with FGFR inhibitors ( $\mathrm{n}=20$, and $80 \%$ treated with BGJ398) had significantly improved OS compared with those who had FGFR2 fusions and were treated with standard therapies ( $\mathrm{n}=34)(\mathrm{P}=0.006)(19)$. IDH targeted therapy using ivosidenib in cholangiocarcinoma was first investigated by Lowery et al. in a phase 1 clinical trial of refractory IDH1mutated cholangiocarcinoma patients. A preliminary median overall survival of 13.8 months was seen (95\% CI, 11.1-29.3) (data censored for 48 patients or 66\%) (20). This signal was further tested in a recently completed randomized phase III study comparing ivosidenib to placebo in the $2^{\text {nd }}$ or $3^{\text {rd }}$-line setting (21). Wainberg et al. reported on a cohort of 33 cholangiocarcinoma patients with BRAF V600E mutations in the ROAR multi-basket study. In this trial, treatment with dabrafenib, a BRAF inhibitor, and trametinib, a MEK inhibitor resulted in a median OS of 11.3 months (95\% CI, 7.3-17.6 months) (22). This myriad of novel agents are offering promise beyond Gem-Cis to 
patients with potentially targetable alterations.

Biliary tract cancers remain one of the most challenging cancers to tackle as we continue to treat these patients with a limited armamentarium. In the palliative setting, systemic therapy with Gem-Cis remains the gold standard for firstline therapy. However, with advances in chemotherapies, genomics, small molecules, and checkpoint inhibitors, an exciting era for treating BTCs is upon us. Promising new regimens such as Gem-Cis-nab-paclitaxel showed improved progression-free survival in a single-arm phase II trial compared with historical controls. The ongoing SWOG 1815 is a pivotal phase III that, if positive, has the potential to establish a new standard of care. New data from the ABC-06 study has shown the benefit of FOLFOX in the $2^{\text {nd }}$ line setting and there are a multitude of other studies in the refractory space that could move the needle forward even more. The current state of therapies in biliary tract offers great promise with numerous different targets and therapies currently being tested in clinical trials. While Gem-Cis continues to be the gold standard for systemic therapy, with many ongoing trials and new drugs in development, there is no doubt that the future is bright for advanced BTCs.

\section{Acknowledgments}

None.

\section{Footnote}

Conflicts of Interest: Shroff RT: Research funding Merck, Halozyme, QED Therapeutics, Exelixis, Pieris Pharmaceuticals; Advisory Board - Merck, QED Therapeutics, Debio Pharmaceuticals, Seattle Genetics, Exelixis. FJ Martinez has no conflicts of interest to declare.

Ethical Statement: The authors are accountable for all aspects of the work in ensuring that questions related to the accuracy or integrity of any part of the work are appropriately investigated and resolved.

\section{References}

1. de Groen PC, Gores GJ, LaRusso NF, et al. Biliary tract cancers. N Engl J Med 1999;341:1368-78.

2. Valle J, Wasan H, Palmer DH, et al. Cisplatin plus gemcitabine versus gemcitabine for biliary tract cancer. $\mathrm{N}$ Engl J Med 2010;362:1273-81.

3. Abdel-Rahman O, Elsayed Z, Elhalawani H. Gemcitabine- based chemotherapy for advanced biliary tract carcinomas. Cochrane Database Syst Rev 2018;4:CD011746.

4. Kang MJ, Lee JL, Kim TW, et al. Randomized phase II trial of S-1 and cisplatin versus gemcitabine and cisplatin in patients with advanced biliary tract adenocarcinoma. Acta Oncol 2012;51:860-6.

5. André T, Reyes-Vidal JM, Fartoux L, et al. Gemcitabine and oxaliplatin in advanced biliary tract carcinoma: a phase II study. Br J Cancer 2008;99:862-7.

6. Sharma A, Dwary AD, Mohanti BK, et al. Best supportive care compared with chemotherapy for unresectable gall bladder cancer: a randomized controlled study. J Clin Oncol 2010;28:4581-6.

7. Lee J, Park SH, Chang HM, et al. Gemcitabine and oxaliplatin with or without erlotinib in advanced biliarytract cancer: a multicentre, open-label, randomised, phase 3 study. Lancet Oncol 2012;13:181-8.

8. Knox JJ, Hedley D, Oza A, et al. Combining gemcitabine and capecitabine in patients with advanced biliary cancer: a phase II trial. J Clin Oncol 2005;23:2332-8.

9. Kim ST, Kang JH, Lee J, et al. Capecitabine plus oxaliplatin versus gemcitabine plus oxaliplatin as first-line therapy for advanced biliary tract cancers: a multicenter, open-label, randomized, phase III, noninferiority trial. Ann Oncol 2019;30:788-95.

10. Shroff RT, Javle MM, Xiao L, et al. Gemcitabine, Cisplatin, and nab-Paclitaxel for the Treatment of Advanced Biliary Tract Cancers: A Phase 2 Clinical Trial. JAMA Oncol 2019;5:824-30.

11. Conroy T, Desseigne F, Ychou M, et al. FOLFIRINOX versus gemcitabine for metastatic pancreatic cancer. $\mathrm{N}$ Engl J Med 2011;364:1817-25.

12. Phelip (PhD): National Library of Medicine (US). 2015 Oct 29 - Identifier NCT02591030. Safety and Efficacy of Modified Folfirinox Versus Gemcis in Bile Duct Tumours. Available online: https://clinicaltrials.gov/ct2/show/ NCT02591030. [cited 2018 October 29].

13. Rogers JE, Law L, Nguyen VD, et al. Second-line systemic treatment for advanced cholangiocarcinoma. J Gastrointest Oncol 2014;5:408-13.

14. Lamarca A, Palmer DH, Wasan HS, et al. ABC-06 I A randomised phase III, multi-centre, open-label study of active symptom control (ASC) alone or ASC with oxaliplatin / 5-FU chemotherapy (ASC+mFOLFOX) for patients (pts) with locally advanced / metastatic biliary tract cancers (ABC) previously-tr. J Clin Oncol 2019;37:abstr 4003.

15. Kim BJ, Yoo C, Kim KP, et al. Efficacy of 
fluoropyrimidine-based chemotherapy in patients with advanced biliary tract cancer after failure of gemcitabine plus cisplatin: retrospective analysis of 321 patients. Br J Cancer 2017;116:561-7.

16. Cai W, Yuan Y, Ge W, et al. EGFR Target Therapy Combined with Gemox for Advanced Biliary Tract Cancers: a Meta-analysis based on RCTs. J Cancer 2018;9:1476-85.

17. Morizane C, Ueno M, Ikeda M, et al. New developments in systemic therapy for advanced biliary tract cancer. Jpn J Clin Oncol 2018;48:703-11.

18. Javle M, Bekaii-Saab T, Jain A, et al. Biliary cancer: Utility of next-generation sequencing for clinical management. Cancer 2016;122:3838-47.

19. Lombardi P, Marino D, Fenocchio E, et al. Emerging molecular target antagonists for the treatment of biliary tract cancer. Expert Opin Emerg Drugs 2018;23:63-75.

Cite this article as: Martinez FJ, Shroff RT. Biliary tract cancers: systemic therapy for advanced disease. Chin Clin Oncol 2020;9(1):5. doi: 10.21037/cco.2019.12.07
20. Lowery (MD): National Library of Medicine (US). 2014 Feb 28. Identifier NCT02073994. Study of Orally Administered AG-120 in Subjects With Advanced Solid Tumors, Including Glioma, With an IDH1 Mutation. Available online: https://clinicaltrials.gov/ct2/show/ NCT02073994. [cited November 2019].

21. Agios Pharmaceuticals, Inc.: National Library of Medicine (US). 2016 Dec 12. Identifier NCT02989857. Study of AG-120 in Previously Treated Advanced Cholangiocarcinoma With IDH1 Mutations (ClarIDHy). Available online: https://clinicaltrials.gov/ct2/show/ NCT02989857. [cited 2019, October 11].

22. Wainberg ZA, Lassen UN, Elez E, et al. Efficacy and safety of dabrafenib (D) and trametinib (T) in patients (pts) with BRAF V600E-mutated biliary tract cancer (BTC): A cohort of the ROAR basket trial. J Clin Oncol 2019;37:abstr 187. 